\title{
Uterine Hemorrhage, CTCAE
}

National Cancer Institute

\section{Source}

National Cancer Institute. Uterine Hemorrhage, CTCAE. NCI Thesaurus. Code C56572.

A disorder characterized by bleeding from the uterus. 